\title{
Desenvolvimento de Recomendações de Acessibilidade e Usabilidade para Ambientes Virtuais de Aprendizagem Voltados para o Usuário Idoso
}

\author{
Michel Kramer Borges de Macedo \\ Universidade Federal de Santa Catarina - UFSC \\ mkbmaced@gmail.com \\ Alice Theresinha Cybis Pereira \\ Universidade Federal de Santa Catarina - UFSC \\ acybis@gmail.com
}

RESUMO: O objetivo deste trabalho foi desenvolver recomendações de acessibilidade e usabilidade para Ambientes Virtuais de Aprendizagem (AVA's) voltados para usuários idosos como estratégia em torná-los acessíveis e de fácil uso aos idosos, que na grande maioria, apresentam declínios de ordem cognitiva, sensorial e física que decorrem da idade. Apesar de nem sempre serem considerados deficientes, estes declínios podem afetar a interação destes com as tecnologias de informação e comunicação, incluindo-se nestas, os Ambientes Virtuais de Aprendizagem, conhecidos por propiciarem uma aprendizagem mais autônoma, dinâmica e personalizada, podem ser uma via promissora para a educação dos idosos. Foi realizada uma pesquisa de campo, de natureza qualitativa, tendo como base a análise da interface da plataforma de Educação a Distância (EaD) - Moodle através da aplicação das técnicas de análise contextual: observação do usuário e grupo focal. Foram traçadas a partir desta pesquisa recomendações de acessibilidade e usabilidade. A intenção destas recomendações é aumentar a facilidade de acesso e uso dos AVA's por idosos, e conseqüentemente, gerar uma procura de cursos à distância mediados por esta tecnologia, possibilitando uma interação entre os idosos e o acesso a novos conhecimentos.

Palavras-chave: Acessibilidade. Usabilidade. Idoso. Ambiente Virtual de Aprendizagem. Educação a Distância.

\section{Development of Recommendations of Accessibility and Usability for Virtual Learning Environment Focused for the Elderly Users}

ABSTRACT: This work aims to develop recommendations of accessibility and usability for Virtual Learning Environment (AVA's) focused elderly users as a strategy to make them accessible and easy to use for the elderly, that in most cases, present declines of cognitive sensory and physical order that elapse from age. Although they are not always considered deficiencies, these declines may affect their interaction with the information and communication technologies, including the Virtual Learning Environment, known by becoming the learning more active, dynamic and personalized could be a new way for the education of the elderly people. It was conducted by field research, qualitative, based on analysis of the interface of the platform Distance Education (EAD) - Moodle by applying the techniques of contextual analysis: observation of the user and focus group. Were possible to be delineated from this research work recommendations for accessibility and usability. The intention of these recommendations is to increase the ease of access and use of the AVA's for elderly people with psychological and physiological declines resulting from these normal ages, and consequently, generates a demand for distance courses mediated by this technology, allowing an interaction between the elderly and the access the new knowledge. 
Keywords: Accessibility. Usability. Elderly. Virtual Learning Environment. Distance Education.

\section{INTRODUÇÃO}

A Organização Mundial de Saúde - OMS considera como idoso todo o indivíduo com idade igual ou superior a sessenta anos. Nos países desenvolvidos, a idade considerada sobe para 65 anos, quando então há necessidade da pessoa receber mais atenção, diante das transformações fisiológicas que começam a se acentuar. A legislação brasileira acompanhou a orientação da OMS estipulando o mesmo limite inicial de idade na Lei 8.842, de 04.01.94, art. $2^{\circ}$. (IBGE, 2007).

No Brasil, o aumento da população de idosos tem promovido mudanças significativas na sua pirâmide populacional. Estas mudanças do perfil da população brasileira são explicadas por especialistas devido à queda da taxa de fecundidade das mulheres, a melhorias das condições nutricionais, o trabalho, o saneamento e a moradia, além de novas descobertas da medicina como antibióticos, vacinas e outros. Isso pode significar menos crianças e mais idosos convivendo no país (IPEA, 2008). O número de idosos na população continuará a aumentar em escala mundial. Na visão de Cuba (2006, p.18), "essa probabilidade alerta para a necessidade de novas alternativas que possam atender, em toda a sua plenitude, os direitos sociais dos idosos brasileiros previstos nas políticas sociais, possibilitando condições dignas a toda a população idosa, no tempo que ainda lhes resta de vida [...]".

Segundo a Síntese dos Indicadores Sociais 2005, divulgada pelo IBGE, com base nos dados da Pesquisa Nacional por Amostra de Domicílios de 2004, o Brasil está entre os primeiros do mundo em população com idade acima de 60 anos. São cerca de 17,6 milhões, que correspondem a 9,7\% da população total (IBGE, 2007a).

Trata-se de um grupo etário altamente heterogêneo, há aquele que continua trabalhando, o recém-saído do seu posto de trabalho, com cerca de 60 anos, até o centenário. Em comum há uma condição de isolamento social e privação de suas demandas.

A aposentadoria representa uma mudança radical, ou seja, ela retira a pessoa do seu convívio dominante do espaço público e passa a experimentar mais intensamente o âmbito privado e doméstico. A aposentadoria pode ser para o idoso um fator de desequilíbrio emocional podendo levá-lo à depressão (Azevedo, 2008). A cada dia mais idosos estão procurando atualizar-se para manter-se ativos tanto socialmente como no mercado de trabalho.

Diversas pesquisas destacam que o idoso tem interesse e possibilidade de conseguir uma boa interação e domínio do computador. Essas pesquisas ressaltam também que este contato pode oferecer alguns benefícios, tais como: melhora na interação social e estímulo mental (Czaja, 1997, Jones; Bayen, 1998, Kachar, 2001).

Percebe-se claramente que nos AVA's falta uma convergência entre acessibilidade e usabilidade em suas interfaces. Estes fatos, na maioria das vezes, impossibilitam ou desestimulam a aprendizagem e a utilização dos usuários, e principalmente do idoso nos AVA's. Com isso, neste artigo pretendeu-se desenvolver recomendações de acessibilidade e usabilidade a serem utilizadas em AVA's voltados aos idosos, aumentando assim a facilidade de acesso e uso por este público e, conseqüentemente, possibilitando à flexibilização na aprendizagem.

\section{FUNDAMENTAÇÃO TEÓRICA}

\subsection{EDUCAÇÃO A DISTÂNCIA E AVA}

Desde a década de 90 com o surgimento da internet, a Educação a Distância vem se aprimorando cada vez mais, através de tecnologias que viabilizam mecanismos de 
comunicação tão eficazes, capazes de suprir a distância geográfica entre aluno, tutor e professor.

A internet, com todos os mecanismos de informação e comunicação, tem proporcionado grandes oportunidades para a $\mathrm{EaD}$, à medida que facilita a troca e a cooperação entre pessoas distantes; assim como possibilita uma comunicação muito mais rápida, intensa e eficiente. Foram introduzidos um grande número de novos recursos, provendo um maior enriquecimento nas comunicações e facilitando a aprendizagem (Valente et. al., 2003).

A EaD tomou um novo impulso com o uso da internet, o que favoreceu a disseminação e a democratização do acesso à educação em diferentes níveis, permitindo atender a grande massa de alunos (Catapan et. al., 2008).

Os avanços e a disseminação do uso das TIC's descortinam novas perspectivas para a $\mathrm{EaD}$ com suporte em Ambientes Virtuais de Aprendizagem acessados via internet, em que a comunicação pode acontecer independente da hora e do lugar e entre todos os participantes do curso.

Behar et. al., (2007) considera AVA como uma plataforma de software (infra-estrutura tecnológica composta pelas funcionalidades e interface gráfica), com a finalidade de promover, complementar, ajudar e facilitar as atividades da educação a distância, efetuando suporte ao desenvolvimento de cursos via Web.

Os AVA's são entendidos como qualquer sistema que dá suporte à aprendizagem na Web. Um AVA é um sistema na Web com necessidades especiais de desenvolvimento: design de interface, planejamento da navegação, desenho do layout e estruturação.

Atualmente, os AVA's estão sendo cada vez mais utilizados no âmbito acadêmico e corporativo como uma opção tecnológica para atender a demanda educacional. Diante disso, destaca-se a importância que sejam respeitados os critérios de usabilidade e acessibilidade no que orienta o desenvolvimento ou o uso desses ambientes, assim oferecendo suporte ao processo ensino-aprendizagem às pessoas independentemente das capacidades físicas, sensoriais e cognitivas. Portanto é possível ressaltar que os AVA's para os idosos devem possuir facilidade na navegação e na localização das informações, diminuindo assim a carga cognitiva.

A aprendizagem mediada por AVA's hoje contribui para uma difusão do conhecimento mais aprazível ao grupo de pessoas portadoras de necessidades especiais, apesar das possíveis barreiras de acessibilidade e usabilidade (Macedo; Ulbricht, 2008). As deficiências mais comuns encontradas nos AVA's são de design de páginas Web, visto que os usuários têm que assimilar primeiramente o projeto visual e navegacional do site antes mesmo de atingir o conteúdo. Os obstáculos que impedem o usuário de realizar suas tarefas vão desde problemas de visualização até dificuldades de acesso a determinados conteúdos.

\subsection{ACESSIBILIDADE NA WEB}

Acessibilidade na Web significa acesso à Web por todos, assim como, pessoas portadoras de necessidades especiais, independente das características do usuário, situação ou ferramenta. A acessibilidade na Web beneficia a todos, inclusive idosos e as pessoas com deficiências.

A acessibilidade na Web envolve sites e sistemas na Web, nos quais as pessoas podem perceber, compreender, navegar e interagir com as informações disponíveis nos mesmos (Macedo; Ulbricht, 2007).

Estão relacionadas abaixo algumas vantagens em tornar os sistemas Web acessíveis:

- Quantidade de usuários com alguma limitação que terão possibilidade de acessar as informações disponíveis: de acordo com a OMS (Organização Mundial de Saúde), 
$10 \%$ da população mundial possui alguma deficiência. No Brasil, o percentual pode chegar a $14,5 \%$ da população.

- É válido dizer que os deficientes são possíveis consumidores, com isso facilitará a aquisição de produtos e serviços pela Web.

- Um portal Web acessível é indexado de forma mais rápida e precisa, pelos mecanismos de busca. Isso faz com que os usuários o localizem com maior rapidez e facilidade. Triacca (2007) refere que quanto melhor a colocação do site, mais visitas ele terá.

- Adotar recomendações de acessibilidade faz com que a página Web seja acessada tanto pelas tecnologias modernas e também pelas mais antigas e pela computação móvel, atingindo um maior contingente de visitantes.

- Vale ressaltar, conforme mostrado no capítulo 2, que os idosos na grande maioria apresentam dificuldades cognitivas e/ou fisiológicas, assim, são considerados portadores de necessidades especiais. A acessibilidade na Web possibilitará o acesso desse grupo de pessoas a Web.

- O cumprimento de medidas legais: a Lei $\mathrm{n}^{\circ}$ 10.098/2000 estabelece normas gerais e critérios básicos para a promoção da acessibilidade às pessoas com deficiência ou mobilidade reduzida (Brasil, 2000). Também, o Decreto $\mathrm{n}^{\circ}$ 5.296/2004, que regulamenta as leis anteriores, versa, pela primeira vez no Brasil, especificamente sobre acessibilidade na internet. Em seu capítulo VI, artigo 47, torna obrigatória a acessibilidade dos portais e sites da administração pública para os usuários deficientes.

\subsection{USABILIDADE}

O termo usabilidade é definido pela norma International Organization for Standardization - ISO 9241 "como a capacidade que um sistema interativo oferece a seu usuário, em determinado contexto de operação, para a realização de tarefas de maneira eficaz, eficiente e agradável" (Cybis et al., 2007, p.14). Desta forma, a usabilidade consiste em uma composição flexível entre aspectos objetivos e subjetivos envolvendo a produtividade na interação. Eficácia significa que o usuário é capaz de realizar a tarefa pretendida, eficiência se refere ao tempo gasto na realização da tarefa e satisfação define o quanto o sistema é aceitável pelos usuários (Cybis et al., 2007).

Conforme Nielsen e Loranger (2007), usabilidade é o termo técnico que referencia a qualidade de uso de uma interface. Quanto maior a facilidade de aprender e memorizar, maior a rapidez de realização de tarefas, menor a taxa de erros e maior a satisfação do usuário, mais usabilidade tem a interface. Bastien et al, (1996) consideram que usabilidade é a capacidade do software em permitir que o usuário alcance suas metas de interação com o sistema.

Para a percepção do usuário de uma boa usabilidade três aspectos são fundamentais na interface: ser de fácil aprendizagem, permitir utilização eficiente e apresentar poucos erros (Nielsen, 1994).

\subsection{USABILIDADE APLICADA NA ACESSIBILIDADE}

Um conceito que está sendo utilizado é o da usabilidade aplicada à acessibilidade. Ao trazer o termo 'usabilidade na acessibilidade', Amstel (2006) refere-se ao princípio básico da Web, que é o acesso por qualquer tipo de pessoa, mas são poucos os projetistas que seguem esse princípio. A maioria dos desenvolvedores de páginas Web ignoram boas práticas que viabilizam o acesso à informação (acessibilidade) e seu uso (usabilidade) por pessoas com necessidades especiais (Amstel, 2006). 
Acessibilidade e usabilidade são conceitos que se inter-relacionam, pois ambos buscam a eficiência e eficácia no uso de uma interface com o usuário.

Entretanto, Acessibilidade é um termo mais genérico já que contempla todos os tipos de usuários e abrange vários aspectos da tecnologia, além de sua interface. Já o conceito de usabilidade engloba aspectos relacionados a interface e a interação de usuários, que não sejam deficientes, com o computador (Miranda, 2002, p.53).

Carrion (2007) distingue: acessibilidade é o termo usado para definir usabilidade para as pessoas com algum tipo de deficiência. Schneiderman (1998) coloca acessibilidade como uma categoria de usabilidade. Já usabilidade é aplicada para que os usuários naveguem sem dificuldades em páginas Web ou consigam um melhor aproveitamento. É definida como a qualidade de interação de uma interface e seu usuário.

Queiroz (2006) complementa referindo que não basta tornar as páginas Web acessíveis, é preciso imergir na lógica da navegação tornando-a mais rápida, fácil e eficiente a todos, para que sua utilização fique fácil e confortável. Dessa forma, segundo o autor, os conceitos de acessibilidade e usabilidade complementam-se com o objetivo de tornar a Web acessível e usável aos usuários.

\section{PROCEDIMENTOS METODOLÓGICOS}

Em relação à natureza da pesquisa, esta é classificada como pesquisa aplicada, gerando, segundo Filho e Santos (2003), conhecimento para aplicação prática em solução de problemas específicos.

Sob o ponto de vista da abordagem do problema, este trabalho caracteriza-se como quanti-qualitativo por sua natureza, pois congrega aspectos quantitativos e qualitativos, visto que o grupo focal e a observação do usuário foram as técnicas de coleta dos dados, os quais foram analisadas em conjunto com o referencial teórico da área.

Para a aplicação das técnicas de análise contextual: grupo focal - GF e observação do usuário, necessitou-se formar um grupo de participantes, composto por quatorze idosos. Destes, 06 são do sexo masculino e 08 do sexo feminino; 12 aposentados e 2 não recebem proventos da previdência; estes com idade média de 66 anos. Todos os participantes possuíam computadores pessoais e acesso a internet por banda larga em suas residências, mas não dominavam esta tecnologia.

O AVA utilizado neste experimento foi o Moodle, o pesquisador instalou sua versão recente em um servidor do HIPERLAB/UFSC, coordenado pela Prof ${ }^{a}$ Alice Theresinha Cybis Pereira, PhD. Realizada a instalação do Moodle, foi criado um curso com o nome de "Tecnologias Computacionais para Melhor Idade" e cadastrado os idosos para participarem do curso.

O Moodle é um sistema complexo, com possibilidade de navegação do usuário por diversas interfaces. O pesquisador apresentou, explicou e fez com que os idosos utilizassem uma a uma das interfaces e ferramentas do Moodle que foram aplicadas às técnicas de GF e Observação do Usuário. Para que a utilização das ferramentas do Moodle se tornasse algo interessante, elas foram introduzidas como forma de promover novos conhecimentos tecnológicos aos idosos, e ao mesmo tempo o pesquisador estava coletando os dados para a pesquisa.

A aplicação das técnicas de análise contextual foram realizadas, no período de março a novembro de 2008. Com um grupo de idosos no horário da manhã e outro à tarde, nas terças e quintas-feiras, das 8 horas às 10 horas e das 14 horas às 16 horas. As reuniões com o grupo focal duravam cerca de 2 horas, orientadas por tópicos referentes aos objetivos traçados para investigação. $\mathrm{O}$ ambiente das reuniões do GF favorecia a 
interação entre os participantes: uma sala com cadeiras confortáveis, computadores atualizados e um ambiente climatizado. Os idosos foram esclarecidos que todas as opiniões interessavam e, portanto, não existiam boas ou más opiniões. Assim, cada membro deveria falar na sua vez, permitindo uma boa gravação das falas e anotações pelo pesquisador. Para registrar as discussões, o pesquisador utilizou um gravador e uma agenda para anotações.

No decorrer das reuniões do GF, o pesquisador apresentava o objetivo da reunião, mantinha o direcionamento das discussões no tópico proposto no início da reunião, evitava a dispersão do grupo, promovendo a participação de todos, eram solicitados exemplos e explicações necessários à compreensão da realidade vivida pelos idosos no AVA.

A Observação dos Usuários foi aplicada quando os idosos estavam utilizando as interfaces e ferramentas do Moodle, em execução de tarefas reais no AVA, em seu ambiente de trabalho, o que pressupõe uma coleta de informações mais abrangente e rica. Eles estavam sendo analisados e monitorados com o objetivo de identificar o comportamento em seu ambiente, os padrões de navegação, as dificuldades de cada indivíduo na interação com o AVA, quais eram suas limitações e o que os impediam na execução de determinadas tarefas.

Ao final da interação dos idosos com o AVA-Moodle o pesquisador apresentava um relatório com suas anotações para os participantes a fim de que eles pudessem efetuar uma "avaliação" ou tecessem alguns comentários sobre os dados coletados.

O pesquisador atuou como mediador e observador na aplicação das técnicas de Grupo Focal e Observação do Usuário.

\section{RESULTADOS DA PESQUISA}

As bibliografias pesquisadas sobre usabilidade e acessibilidade, juntamente com a análise dos resultados da aplicação das técnicas de Grupo Focal e Observação do Usuário, possibilitaram o desenvolvimento de um conjunto de recomendações para nortear a interface do AVA - Moodle. Conforme o grau do problema e a intensidade ocorrida na utilização do AVA pelos idosos, constituíram-se as recomendações para solucioná-los.

Tais recomendações foram numeradas para facilitar sua referência, mas a ordem das mesmas não implica em um critério de prioridade ou importância em relação às outras.

As recomendações que provém desta pesquisa fundamentam-se em comentários dos idosos, na análise dos dados resultantes da aplicação das técnicas de grupo focal e observação do usuário, e na bibliografia pesquisada.

Recomendação $\mathbf{n}^{\mathbf{0}}$ 01: Manter bom contraste entre as cores de fundo da página e o texto, evitando cores de fundos muito brilhantes.

Cybis et al., (2007) sugerem em seu livro que as interfaces na Web devem aparecer com letras claras sobre um fundo escuro para pessoas com problemas visuais ou os idosos, o fundo brilhante pode ofuscar completamente as letras escuras.

Na utilização do AVA todos os idosos tiveram dificuldades em perceber o texto quando a cor de fundo da página Web estava muito brilhante. É recomendado utilizar um contraste favorável entre as cores do fundo da página com as cores do texto, usando preferencialmente cores escuras para o fundo da página e cores claras para letras.

Comentário dos idosos: "Eu não estou conseguindo visualizar a informação, você que é jovem poderia me dizer o que está escrito aqui na tela? Há muito brilho e está ofuscando minha visão". 
Recomendação $\mathbf{n}^{\mathbf{0}}$ 02: Evitar a utilização de links no meio de conteúdo que promovam a abertura de muitas janelas.

Os idosos ao abrirem vários links em novas janelas ficavam totalmente desorientados na navegação pelo AVA. É recomendado que se utilize no máximo um link a ser aberto em uma nova janela (janela secundária). Utilizar links que se abram em novas janelas acarreta em um aumento na carga perceptiva e cognitiva do idoso. O idoso ao utilizar o computador com várias janelas abertas acaba fechando-as.

Comentário dos idosos: "Nossa!! Como tem janelas abertas. Fui eu que fiz isso!!! Eu não estou conseguindo achar meu texto inicial. Onde será que o coloquei hehe!!"

A pesquisa bibliográfica ajuda a confirmar a recomendação. Cybis et al., (2007) colocam que deve ser respeitada a capacidade de trabalho perceptivo, cognitivo e motor do idoso. Ao abrir várias janelas o usuário terá um conjunto de ações maiores para alcançar sua meta ou realizar uma tarefa. Ao diminuir o número de páginas que o usuário deve acessar, ele estará diminuindo a carga de trabalho e a probabilidade de ocorrências de erros.

Recomendação nº 03: Escrever o texto na linguagem do usuário.

$\mathrm{O}$ idoso tem um sério problema de não compreender a informação se ela não estiver colocada de uma forma clara e na voz ativa. Recomenda-se utilizar o texto na linguagem do usuário e ser escrito de forma afirmativa e direta, na voz ativa, evitando pontuações desnecessárias e apresentando argumentos segundo uma ordem lógica. A utilização de uma linguagem clara e simples proporciona uma comunicação eficaz.

O AVA deve utilizar palavras, frases e conceitos familiares, ao invés de termos técnicos. As convenções do mundo real devem ser seguidas, fazendo com que as informações apareçam em uma ordem lógica e natural ao usuário (Dias, 2007).

Os textos contidos no AVA devem ser escritos de maneira clara, direta e objetiva, excluindo o emprego da voz passiva. Com a idade o idoso perde a habilidade de compreender um texto. Essas mudanças geralmente não são dramáticas (Dias, 2007).

Comentário dos idosos: "Nós lemos três vezes o texto, mas ele não nos parece objetivo, isso dificulta e muito nosso entendimento".

Recomendação $\mathbf{n}^{\mathbf{0}}$ 04: Manter espaçamento duplo entre as linhas de um texto.

O espaçamento entre as linhas de um texto para leitura dos idosos deve ser duplo, pois o uso de um espaço adequado entre as linhas favorece a realização de movimentos oculares rápidos para os saltos entre o final de uma linha e o início da próxima.

Comentário dos idosos: "Como tenho dificuldades em realizar uma leitura no AVA!! Quando o espaçamento entre o texto está simples, dificulta a leitura, pois estou finalizando a leitura e tenho que movimentar meus olhos para o início da próxima linha. Por estarem muito próximas acabo me perdendo. Uma maior distância entre as linhas facilita minha leitura".

Recomendação $\mathbf{n}^{\mathbf{0}}$ 05: Usar tamanho da fonte para textos maior que 12 pontos.

Textos com fontes menores que 12 pontos dificultam muito a leitura dos idosos. Recomenda-se utilizar o tamanho da fonte nos textos de no mínimo 12 ou 14 pontos ou grande o suficiente para ser lida por pessoas idosas, pois pessoas com a vista enfraquecida necessitam de letras maiores para uma leitura adequada.

Comentário dos idosos: "Acho que está na hora de trocar as lentes do meu óculos! Não estou conseguindo ler texto na tela do computador em tamanho de 10 pontos. Ah! um tamanho de 12 ou 14 pontos facilita e muito nossa leitura". 
Recomendação no 06: Manter texto alinhado à margem esquerda.

$\mathrm{O}$ alinhamento do texto deve feito pela margem esquerda, pois favorece a leitura por criar espaços uniformes entre as palavras e por permitir saltos entre o final de uma linha e o início da próxima linha (CYBIS et al., 2007).

É recomendado utilizar o texto alinhado à margem esquerda para favorecer a orientação na leitura do texto.

Comentário dos idosos: "O texto alinhado pela margem esquerda facilita nossa leitura. As linhas não terminam no mesmo local alinhada na margem direita".

Recomendação $\mathbf{n}^{\mathbf{0}}$ 07: Usar ícones grandes associados às legendas.

Cybis et al. (2007) recomendam ao utilizar ícones em uma interface, ter o cuidado deste ser simples, com poucos elementos e poucas cores, de modo a permitir a identificação e compreensão das funções dos ícones pelos usuários.

A percepção dos ícones pode ser um incômodo e às vezes impossível de ser realizada por idosos com problemas de visão. Para facilitar a identificação e compreensão das funções dos ícones, as imagens devem ser ampliadas e estar associadas a etiquetas textuais.

Recomenda-se utilizar ícones grandes, legíveis, significativos, facilmente discriminados e rotulados.

Comentário dos idosos: "Estou mal conseguindo ver esse ícone, não consigo associá-la a nenhuma função no AVA. Você poderia me dizer qual é a imagem existente e a função?”.

Recomendação $\mathbf{n}^{\mathbf{0}}$ 08: Dispor as informações de forma hierárquica nas áreas mais percebidas.

Recomenda-se que as informações mais importantes devem se encontrar nas zonas freqüentemente percorridas pelos olhos na tela, no canto superior esquerdo, se a tela contém numerosas informações e, na parte central, se a tela não tem muitas informações.

Comentário dos idosos: "Não consigo achar o link que o professor solicitou. Ah! Ele estava no final da página Web ao lado direito. Eu estava procurando-o no canto superior esquerdo e na parte central".

Recomendação nº 09: Dispor mapa de navegação.

O mapa do site possibilita um aumento na probabilidade de um usuário encontrar o que está procurando, representa um importante recurso na diminuição da desorientação, que é um dos problemas que mais afetam os usuários em ambientes extensos e repletos de todo o tipo de informação (Dias, 2007).

Deve-se utilizar um mapa de navegação no AVA que apresente como ele é organizado. O mapa do site mostra um panorama da organização do site.

Comentário dos idosos: "Com o mapa de navegação do site fica muito fácil encontrar a informação que desejamos".

Recomendação no 10: Usar ícones grandes associados às legendas.

Cybis et al. (2007) recomendam ao utilizar ícones em uma interface, ter o cuidado deste ser simples, com poucos elementos e poucas cores, de modo a permitir a identificação e compreensão das funções dos ícones pelos usuários.

A percepção dos ícones pode ser um incômodo e às vezes impossível de ser realizada por idosos com problemas de visão. Para facilitar a identificação e compreensão das 
funções dos ícones, as imagens devem ser ampliadas e estar associadas a etiquetas textuais.

Recomenda-se utilizar ícones grandes, legíveis, significativos, facilmente discriminados e rotulados.

Comentário dos idosos: "Estou mal conseguindo ver esse ícone, não consigo associá-la a nenhuma função no AVA. Você poderia me dizer qual é a imagem existente e a função?”.

\section{CONCLUSÕES}

A possibilidade de flexibilização na aprendizagem e a necessidade de formação continuada dos idosos adicionada a facilidade de desenvolvimento de sistemas na Web, fazem com que os Ambientes Virtuais de Aprendizagem se tornem um nicho atrativo a ser explorado por idosos.

A utilização das novas tecnologias abre novas perspectivas de formação e integração social, garantindo o acesso à informação de modo a não penalizar as pessoas com necessidades especiais, a exemplo dos idosos. Para o grupo de pessoas idosas as interfaces devem ser acessíveis e usáveis, elaboradas de forma a suprir as suas limitações.

Para que os cursos a distância via Web se tornem acessíveis a um público muito maior, os AVA's quando desenvolvidos deverão seguir as recomendações de acessibilidade e usabilidade para que as pessoas portadoras de necessidades especiais possam acessar o conteúdo na Web e compreendê-lo.

Ao avaliar as interfaces do Ambiente Virtual de Aprendizagem - Moodle percebeu-se que as deficiências dos idosos não são o real problema, mas sim as tecnologias utilizadas, pois elas não oferecem a possibilidade de utilização eficiente por esse grupo de pessoas. Assim, as recomendações de usabilidade e acessibilidade podem promover a utilização eficiente dos AVA's pelos idosos.

Este trabalho enriqueceu a discussão sobre a utilização de AVA's acessíveis e usáveis em cursos de educação a distância na formação continuada de idosos.

\section{REFERÊNCIAS}

AMSTEL, Frederick Van. Usabilidade na acessibilidade. 2006. Disponível em: $<$ http://www.usabilidoido.com.br/usabilidade_na_acessibilidade.html $>$. Acesso em: 15 jan 2009.

AZEVEDO, João R. D. A Ansiedade na Terceira Idade. Disponível em:

$<$ http://www.boasaude.com/lib >. Acesso em: 06 nov. 2008.

BASTIEN, C; SCAPIN, D.L.; LEULIER, C. (1996). Looking for Usability Problems With the Ergonomic Criteria and the ISO 9241-10 dialogue priciples, In.

Proceedings of CHI'96. Vancouver. Canada.

BEHAR, Patricia Alejandra, et. al. Avances en Sistemas e Informática, Vol. 4 No. 1 Junio de 2007, Medellín, ISSN 1657-7663

CARRION, W. Acessibilidade Web. Disponível em:

$<$ http://imasters.uol.com.br/artigo/3134/acessibilidade/acessibilidade_web/>. Acesso em: 12 jan. 2009.

CATAPAN, Araci Hack; et. al. Introdução a educação a distância.

Florianopolis: Biologia/EAD/UFSC, 2008. 122p.

CUBA, Conceição de Maria G. Braga. Ninguém Vive Sem Amizade! A Importância da Amizade Política dos Idosos Colaboradores da UnATI/UERJ. 2006. 255 f.

Dissertação (Mestrado) - Departamento de Serviço Social, Pontifícia Universidade 
Católica do Rio de Janeiro, Rio de Janeiro, 2006. Disponível em:

$<$ http://www.maxwell.lambda.ele.puc-rio.br>. Acesso em: 18 de set. 2008.

CYBIS, Walter; BETIOL, Adriana Holtz; FAUST, Richard. Ergonomia e Usabilidade:

Conhecimentos, Métodos e Aplicações. São Paulo: Novatec, 2007. 344p.

DIAS, Claudia. Usabilidade na Web: Criando portais mais acessíveis. Rio de Janeiro: Alta Books, 2007. 296p.

FILHO, Domingos Parra; SANTOS, João Almeida. Metodologia Científica. São

Paulo: Futura, 6a Ed. 2003. 277p.

IBGE - Instituto Brasileiro de Geografia e Estatística. Dia do Idoso. Disponível em:

$<$ http://www.ibge.gov.br/ibgeteen/datas/idoso/idoso_no_mundo.html> . Acesso em: 04 de set. 2007.

Cresce a presença da população com 50 anos ou mais no mercado de

trabalho. Disponível em:

$<$ http://www1.ibge.gov.br/home/presidencia/noticias/noticia_visualiza.php?id_noticia= 648\&id_pagina=1>. Acesso em: 12 out. 2007a.

IPEA - Instituto de Pesquisa Econômica Aplicada. Como vai População Brasileira.

Ano IV número 2. Disponível em:

<http://www.ipea.gov.br/pub/comovai/comovai.html>. Acesso em: 02 set. 2008.

KACHAR, Vitória. A terceira idade e o computador: interação e produção no ambiente educacional interdisciplinar. Tese de doutorado defendida no Programa de Pós-Graduação em Educação: Currículo da PUC. São Paulo: PUC-SP, 2001.

MACEDO, Claudia Mara Scudelari; ULBRICHT, Vânia Ribas. CONSIDERAÇÕES DE ACESSIBILIDADE EM EDUCAÇÃO A DISTÂNCIA. Congresso Nacional de Hipermídia para Aprendizagem - $3^{\circ}$ CONAHPA, 2008, São Paulo: Anhembi Morumbi, 2008.

MIRANDA, Andréa da Silva. Recomendações de acessibilidade digital em cursos de educação básica a distância via web para portadores de deficiência visual.

Florianópolis, 2002. 160 f. Dissertação (Mestrado) - . Programa de Pós-Graduação em Ciência da Computação. UNIVERSIDADE FEDERAL DE SANTA CATARINA, Florianópolis, 2002. Disponível em: <http://www.tede.ufsc.br/teses/PGCC0501.pdf>. Acesso em: 02 jun. 2008.

NIELSEN, Jakob; LORANGER, Hoa. Usabilidade na Web - Projetando Websites com Qualidade. Rio de Janeiro: Campus. 2007. 432p.

NIELSEN, Jakob. Enhancing the Explanatory Power of Usability Heuristics.

Proceeding of ACM CHI' 94 Conference on Human Factors in computing Systems.

Pp.152-158, v.1, 1994.

QUEIROZ, Marco Antonio de. Acessibilidade web: tudo tem sua primeira vez.

Bengala digital. 2006. Disponível em:

$<$ http://www.bengalalegal.com/capitulomaq.php>. Acesso em: 5 dez. 2009.

SHNEIDERMAN, Ben. Designing the user interface: Strategies for effective

Human-Computer Interaction. 3 edition. Berkeley, California: Addison Wesley

Longman, Inc., 1998.

TRIACCA, Anderson. Google, o grande cego. MXStudio. Disponível em: <http://www.mxstudio.com.br/dreamweaver/google o grande cego/>. Acesso em: 10 jan. 2007.

VALENTE, J. A.; PRADO, M. E. B.; ALMEIDA, M. E. B. Educação a Distância Via Internet. São Paulo: Avercamp, 1. ed. 2003. 